\title{
Numerical Investigation and Multi-Objective Optimisation of the Aerodynamics of a Hyperloop Pod
}

\author{
$\underline{\text { Nivedan Vishwanath }}^{1}$, Tushar Maloo ${ }^{2}$, Prithvi Ramesh ${ }^{2}$, Dheeraj Agarwal ${ }^{3}$ \\ ${ }^{1}$ Student, Department of Mechanical Engineering, BITS Pilani, Hyderabad Campus, Hyderabad, India \\ f20181005@hyderabad.bits-pilani.ac.in \\ 2 Student, Department of Mechanical Engineering, BITS Pilani,K K Birla Goa Campus, Goa, India \\ f20180848@goa.bits-pilani.ac.in; f20180502@goa.bits-pilani.ac.in \\ ${ }^{3}$ Lecturer, School of Engineering, University of Liverpool, United Kingdom \\ Dheeraj.Agarwal@liverpool.ac.uk
}

\begin{abstract}
Hyperloop systems are stated to be the fifth mode of transportation and would have the capacity to ferry passengers at ground speeds of over $1000 \mathrm{~km} / \mathrm{h}$. The transonic operating conditions of hyperloop pods make them susceptible to flow choking, thus demanding careful aerodynamic design. In this study we carry out the aerodynamic optimisation of a Hyperloop pod structure. A preliminary two-dimensional steady state computational fluid dynamics analysis of the pod is done to establish a baseline. In the optimisation phase, the multi-objective genetic algorithm is used to evaluate the lowest possible drag and lift coefficients. As a result of the optimisation study, we were able to reduce the drag coefficient by $22.44 \%$ and the lift coefficient by $14.87 \%$ as compared to the initial design of the pod.
\end{abstract}

Keywords: Hyperloop, Multi-Objective Optimisation, MOGA, Aerodynamics, High Speed

\section{Introduction}

Rising environmental concerns have led people to shift from cars to public transportation systems. In order to account for the large influx of new users, existing mass transportation systems are required to be very efficient in ferrying a large number of people in less time. Currently, the high-speed rail systems are capable to reach a top speed of approximately $500 \mathrm{~km} / \mathrm{h}$, however they operate at much lower speeds of $\sim 300 \mathrm{~km} / \mathrm{h}$. This could be attributed to many reasons: at high speeds the contact friction between the rail and the wheels produce excessive heat, thus limiting their performance. Moreover, since trains currently operate at ambient conditions, the form drag increases rapidly at high speeds. Maglev trains, which operate on the principle of magnetic levitation [1, 2], are able to address the issue associated with friction heating, as they do not make contact with rails, however the limitations of pressure drag at high speeds still exists. These operational limitations of current vehicles motivated the research and development of hyperloop systems [6].

A hyperloop consists of pods that can be accelerated in partially evacuated tubes to over $1000 \mathrm{~km} / \mathrm{h}$. They are magnetically levitated on a central rail and are propelled using Linear Induction Motors (LIM). Inside the tube, the low-pressure environment ensures low air density, leading to lower form drag than vehicles travelling at similar speeds in ambient conditions. This combined with the absence of contact friction, would enable operational speeds well beyond the capability of the existing railway systems. The hyperloop is planned to provide point to point connections at speeds resembling air travel. Maintaining efficiency at high operating speeds of $M_{\text {operational }} \sim 0.9$ would require an efficient aerodynamic design. While the pressure inside the tube is nearly $0.1 \%$ of the atmospheric pressure [6], it is necessary to carefully model the remaining air. Since transonic flow in confined tubes produce complex flow structures, it is difficult to simulate the phenomenon with high fidelity.

Due to low operating pressure inside the tube, the Reynolds number $(\mathrm{Re})$ of the current flow is $\mathscr{O}\left(10^{5}\right)$. This makes it susceptible to laminar to turbulent transition [7]. Since the flow can become locally supersonic over certain regions of the pod, they can also trigger boundary layer separations [8]. All of these factors would contribute to increase in drag, apart from the drag caused due to the choked flow [3].

The flow becomes choked at the point of minimum cross-sectional area between the pod and the tube and when it occurs, the mass flow rate cannot increase downstream, even if the pressure difference in the downstream is increased. There are two ways to ensure that the pod does not violate the Kantrowitz limit [5]. The first option is to decrease the blockage ratio, which is the ratio of cross sections of the pod and the tube $\left(B_{r}=\mathrm{A}_{\text {pod }} / \mathrm{A}_{\text {tube }}\right)$. The other option is to introduce a compressor in front of the pod that can redirect excess mass flow rate to the back of the pod even when the $B_{r}$ is high. While this option was initially proposed in the original introduction of the Hyperloop, it is difficult to simulate the aerodynamics of the pod in the presence of a compressor. 
The objective of this study is to develop an aerodynamic shape optimisation framework for a Hyperloop pod, taking into consideration the drag coefficient and the Kantrowitz limit. This is realised by performing two-dimensional numerical simulations using ANSYS Fluent, coupled with the ANSYS DesignXplorer optimisation interface. The optimisation routine controls the geometry using Bezier control points and produces a geometry that minimises the drag force while staying within the Kantrowitz limit. The baseline pod geometry is designed in accordance with the minimum geometry requirements set by Hyperloop India, a student team at BITS Pilani, India.

This paper is structured as follows: section 2 outlines the numerical methods employed in this work, followed by the problem definition and computational methodology in section 3. section 4 provides detailed results for the developed optimisation framework, followed by the conclusions in section 5 .

\section{Numerical Methodology}

We carry out a Goal-Driven Optimisation (GDO) study based on the Multi-Objective Genetic Algorithm (MOGA) formulation. It is a hybrid variant of the Non-dominated Sorted Global Algorithm-II (NSGA-II) based on controlled elitism concepts. The constraint handling uses the same non-dominance principle as the objective functions, thus ensuring that the feasible solutions are always ranked higher than the infeasible ones. MOGA generates new population sets using cross-over and mutation. Cross-over combines two chromosomes (also called parents) to produce a new chromosome, such that it can be better than both the parents and take the best characteristics from both the parents. Two parent vectors are linearly combined to produce a new offspring. During mutation, one or more gene values in a chromosome are altered so that the algorithm is able to arrive at a better solution as compared to the previous iterations.

The objective functions for the MOGA algorithm are the minimisation of the coefficient of drag $\left(C_{D}\right)$ and the coefficient of lift $\left(C_{L}\right)$. In addition to drag minimisation, we also minimise the lift to prevent the pod from taking off the ground unevenly. The input parameters are the $y$-coordinates of the 12 control points that make up the pod spline.

$$
y_{1}, y_{2}, \ldots y_{12} \in \text { Control Points }
$$

The constraint functions are specified as bounds to the control points - each control point is bounded between a highest possible value $\left(H_{n}\right)$ and a lowest possible value $\left(L_{n}\right)$, such that the pod's overall shape is not altered. Therefore, the constraints are of the form:

$$
L_{n} \leq x_{n} \leq H_{n}, n \in(1,2, . ., 12)
$$

The spatial constraints in our problem are governed by the Kantrowitz limit, which is calculated using the following relation [5],

$$
\frac{A_{\text {flow }}}{A_{\text {tube }}}=M_{0}\left(\frac{\gamma+1}{2}\right)^{\frac{\gamma+1}{2(\gamma-1)}} \times\left(1+\frac{\gamma-1}{2} M_{0}^{2}\right)^{-\frac{\gamma+1}{2(\gamma-1)}}
$$

The values of $\mathrm{L}$ and $\mathrm{H}$ are constants defined for each control point. To retain the pod's shape, the control points maintain their relative position as follows:

- For the nose section of the pod $\left(y_{1}, y_{2}, \ldots y_{6}\right)$, the ranges represented by the bounds are mutually exclusive for those control points, that is, $L_{n}>H_{n-1}$.

- For the control points of the pod $-y_{7}, y_{8}, \ldots y_{12}$, the shape has slight upward incline that reaches a maximum height and then has a considerable downward incline. For this region the ranges represented by the bounds are not mutually exclusive as the upward and downward inclines are not very steep. Here the parameter relations are as follow:

\begin{tabular}{|c|c|c|}
\hline Left Expression & Operator & Right Expression \\
\hline$y_{6}$ & $\leq$ & $y_{7}$ \\
\hline$y_{7}$ & $\leq$ & $y_{8}$ \\
\hline$y_{8}$ & $\geq$ & $y_{9}$ \\
\hline$y_{9}$ & $\geq$ & $y_{10}$ \\
\hline$y_{10}$ & $\geq$ & $y_{11}$ \\
\hline$y_{11}$ & $\geq$ & $y_{12}$ \\
\hline
\end{tabular}

Table 1: Constraint relations on the pod geometry

- The point $y_{8}$ is the topmost point of the pod, whose upper bound $H_{n}$ is calculated from the Kantrowitz limit relation. 
- The point $y_{12}$ is the bottom most point of the main body of the pod, whose lower bound $L_{n}$ is set according to the geometric constraints imposed by the minimum volume requirement of the pod.

\section{Computational Method}

We study the transonic flow aerodynamics of a Hyperloop pod for multiple design configurations. The objective is to converge to a design that experiences the least drag and lift force during its motion through the tube. A baseline pod of length $(\mathrm{L})$ of $3.5 \mathrm{~m}$ and a height $(\mathrm{H})$ of $0.355 \mathrm{~m}$ is considered. The values of $\mathrm{L}$ and $\mathrm{H}$ are in accordance to the designs made by Hyperloop India, a student team at BITS Pilani. The flow domain is modelled according to a scaled-down version (1:100) of the test tube at the SpaceX site and has a height of $1.4 \mathrm{~m}$. The upstream and downstream lengths are taken as $5 \mathrm{~L}$ and $10 \mathrm{~L}$, respectively. The blockage ratio $\mathrm{Br}_{\mathrm{i}}$ is defined as the ratio of the projected area of the pod to the height of the computational domain. $\mathrm{Br}_{\mathrm{i}}$ for this baseline geometry is computed to be 0.253 . Figure 1 represents the schematic of the computational domain. The permissible pod velocity, as calculated from equation 1 is Mach 0.5 , for which the flow around the pod is exactly sonic. For our study, we have considered the pod velocity to be equal to $163.2 \mathrm{~m} / \mathrm{s}$, corresponding to $M=0.476$.

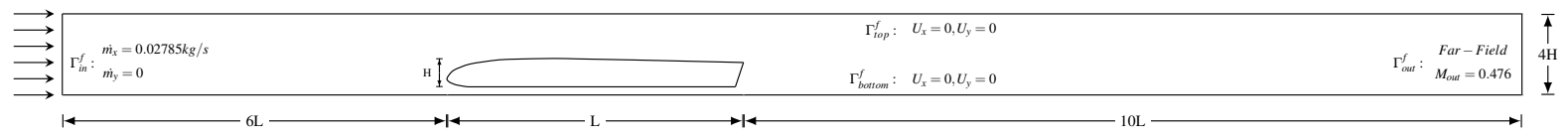

Figure 1: Schematic of 2D computational domain with boundary conditions

The flow is assumed to be steady, inviscid, and adiabatic. Since the current case dealt with accelerating flows, entropy was considered to be a constant. [4] The flow resides in the continuum regime, since the Knudsen number $\left(K_{n}\right)$ is of the order $10^{-6}$.

The $k-\omega$ SST RANS model is used for the turbulence modelling. A constant mass-flow rate enters the domain at the inlet, with a pressure far-field condition imposed at the outlet. All the walls in the domain are non slipping, to simulate the pod motion through the tube. An operating pressure of $1000 \mathrm{~Pa}$ is considered.

A grid independence study is performed to determine the most optimum grid for the subsequent simulations. Four cases with increasing mesh refinement were simulated and the force coefficients were compared as shown in Table 2.

\begin{tabular}{|c||c|c|c|c|}
\hline Mesh No: & Mesh 1 & Mesh 2 & Mesh 3 & Mesh 4 \\
\hline Mesh Count & 225340 & 345238 & 547008 & 1136185 \\
\hline$C_{d}$ & 1.4206 & 1.4116 & 1.4116 & 1.4114 \\
$C_{l}$ & 6.8800 & 6.8387 & 6.8374 & 6.8423 \\
\hline
\end{tabular}

Table 2: Results from the mesh convergence study carried out to ensure grid independence of the solution for the case of flow past the Hyperloop pod at $\operatorname{Re}=3.89 \times 10^{5}$.

For the subsequent optimisation study, Mesh 2 was chosen because the percent change in the drag and lift coefficient values was less than $0.02 \%$ between Mesh 2 and Mesh 3.

\section{Results}

The optimisation algorithm is run for 85 design points, generated from the constraints entered for the 12 spatial coordinates that make up the pod geometry. The MOGA routine is such that it outputs the global extrema for the set of constraints provided for the design variables. Figure 2 compares the initial and final design parameters obtained from the simulation. The nose radius of the optimised geometry is marginally lesser than that of the baseline geometry. There is also a slight reduction in the overall height of the pod, which would allow more mass rate to pass around the pod at any given point in time. 


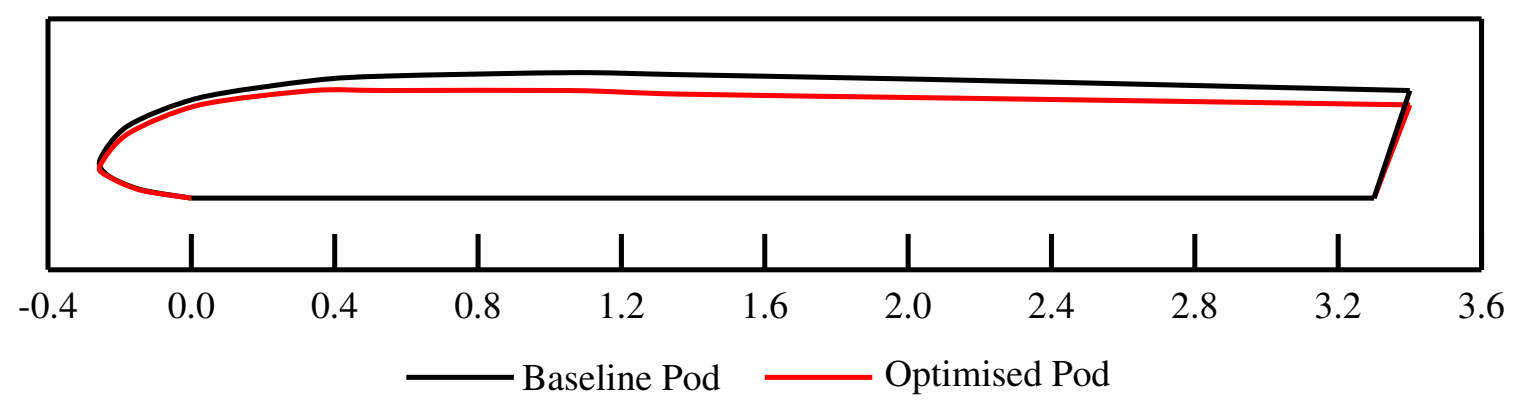

Figure 2: Comparison of baseline and optimised pod geometry

The optimised geometry is simulated to obtain the values of aerodynamic coefficients. Table 3 compares the force coefficients of the baseline geometry with the optimised one. We can observe that the drag coefficient reduced by around $22 \%$ along with a $15 \%$ decrease in the lift coefficient. This decrease in the aerodynamic coefficient values clearly indicate an improvement in the aerodynamic flow characteristics as a result of the optimisation step.

\begin{tabular}{|c||c|c|c|}
\hline Case: & Baseline Geometry & Optimised Geometry & \% Change \\
\hline$C_{d}$ & 1.4113 & 1.0946 & -22.44 \\
$C_{l}$ & 6.8362 & 5.8196 & -14.87 \\
\hline
\end{tabular}

Table 3: Summary of force coefficients obtained from 2D simulations of the baseline and the optimised pod geometry

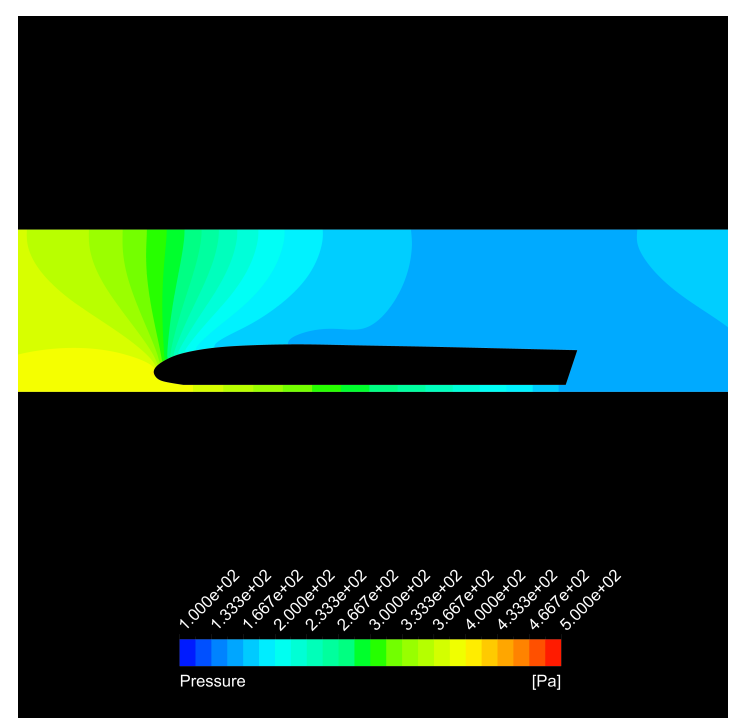

(a) baseline pod

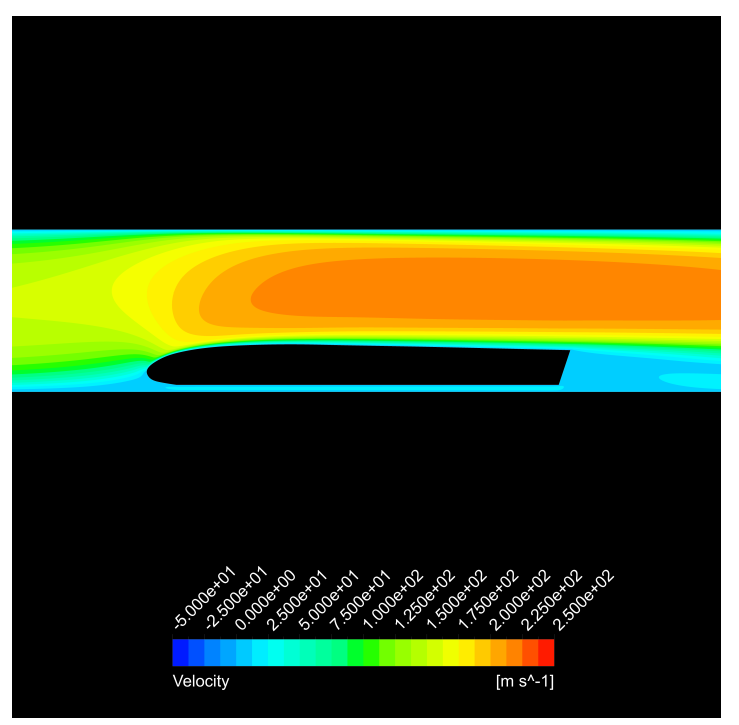

(b) baseline pod

Figure 3: Pressure(a) and Velocity (b) contours for the baseline pod 


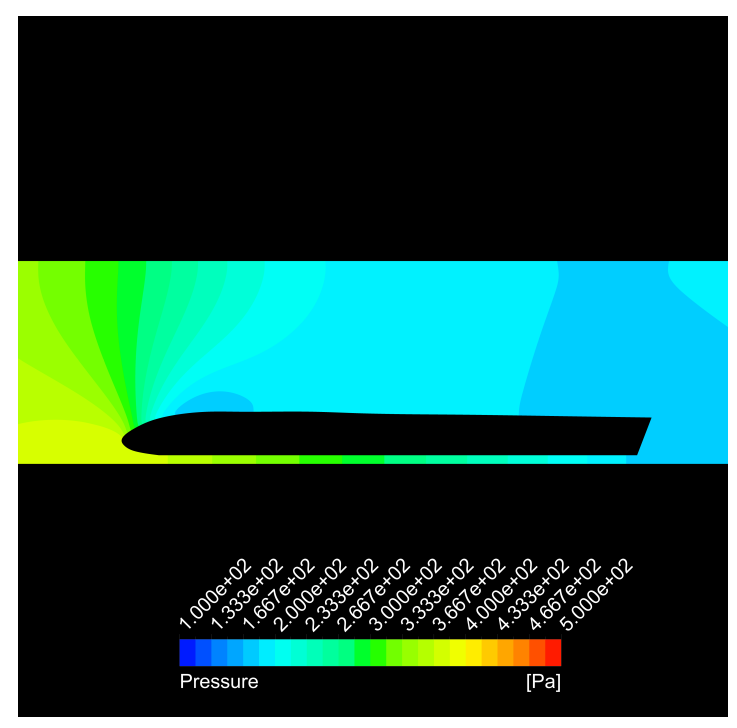

(a) optimised pod

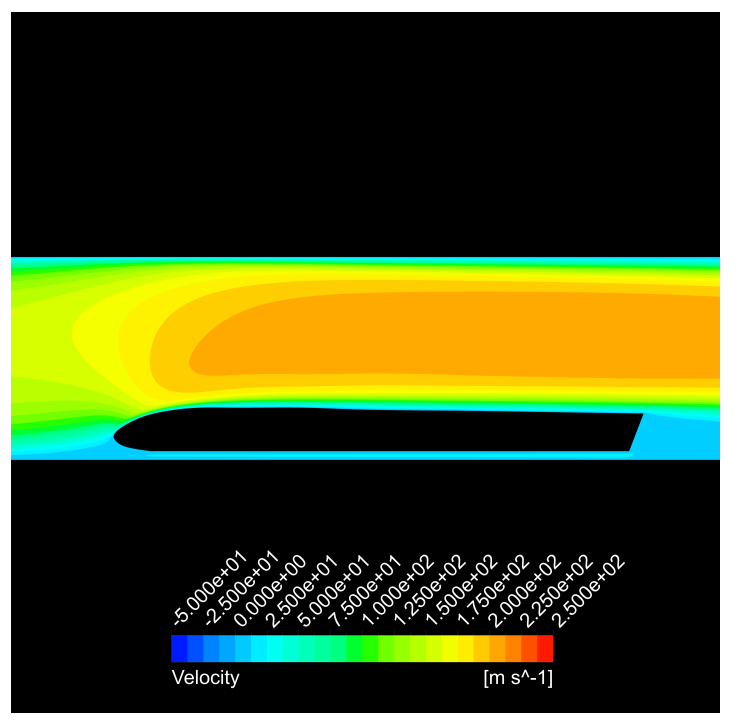

(b) optimised pod

Figure 4: Pressure(a) and Velocity (b) contours for the optimised pod

Figures 3 and 4 present the pressure and velocity contours for the baseline and optimised configurations respectively. The contours for both the configuration are plotted on a common range to visualise the changes effectively. Upon observation, it was found that the flow velocity over the pod for the optimised case is lower than that of the baseline case. This indicates that the pressure difference between the forward and rear portions of the pod is lesser in case of the optimised case. A lower pressure difference means that lesser drag is experienced by the pod as it moves through the tube.

\section{Conclusion}

The current study focused on the aerodynamic optimisation of a Hyperloop pod using multi-objective global optimisation algorithm. The optimisation environment was created on ANSYS and simulations were run for 85 different configurations. The results from the simulations demonstrate the fact that the optimised configuration of the pod would be aerodynamically efficient as compared to the baseline, while adhering to the minimum volume limit to safely pack all internal components inside the pod. Future studies can focus on geometries in which the flow is predominantly choked. That would test the robustness of the routine to find the best possible geometries.

\section{Acknowledgements}

The authors would like to thank the Department of Mechanical Engineering, BITS Pilani, K.K. Birla Goa Campus for providing the required computational facilities.

\section{References}

[1] H.W. Lee, K.C. Kim, and J. Lee. Review of maglev train technologies. IEEE Transactions on Magnetics, 42(7):1917-1925, 2006.

[2] L. Yan. Development and Application of the Maglev Transportation System. IEEE Transactions on Applied Superconductivity, 18(2):92-99, 2008.

[3] J. Braun, J. Sousa, and C. Pekardan. Aerodynamic Design and Analysis of the Hyperloop. AIAA Journal, 55(12):4053-4060, 2017.

[4] R. Fox, A. McDonald, and P. Pritchard, Introduction to Fluid Mechanics, 8th ed., 10, Vol. 4. John Wiley \& Sons, Inc., 2011.

[5] D.M. Van Wie, F.T. Kwok, R.F. Walsh. Starting characteristics of supersonic inlets. 32nd joint propulsion conference and exhibit., p.2914, 1996.

[6] E. Musk. Hyperloop Alpha. https://www.tesla.com/sites/default/files/blog_images/hyperloop-alpha.pdf. [Online; accessed 14-June-2021]. 
[7] M. Opgenoord and P. Caplan. On the Aerodynamic Design of the Hyperloop Concept. AIAA Journal, 2016.

[8] H. Schlichting, and K. Gersten. Boundary Layer Theory, 9th ed. Springer-Verlag, 2017. 